\title{
Correction to: Streaming active learning strategies for real-life credit detection: assessment and visualization
}

\author{
Fabrizio Carcillo ${ }^{1}$ - Yann-Aël Le Borgne ${ }^{1}$. Olivier Caelen ${ }^{2} \cdot$ Gianluca Bontempi ${ }^{1}$
}

Published online: 2 May 2018

(c) Springer International Publishing AG, part of Springer Nature 2018

\section{Correction to: International Journal of Data Science and Analytics \\ https://doi.org/10.1007/s41060-018-0116-z}

A line was misplaced in Table 1 . The correct Table 1 is given below. The original article has been corrected.

The original article can be found online at https://doi.org/10.1007/ s41060-018-0116-z.

\footnotetext{
Fabrizio Carcillo

fabrizio.carcillo@ulb.ac.be

Yann-Aël Le Borgne

yleborgn@ulb.ac.be

Olivier Caelen

olivier.caelen@worldline.com

Gianluca Bontempi

gbonte@ulb.ac.be

1 Machine Learning Group, Computer Science Department, Faculty of Sciences, Université Libre de Bruxelles (ULB), Brussels, Belgium

2 R\&D, Worldline, Brussels, Belgium
} 
Table 1 Summary of active learning and semi-supervised strategies described in the paper

\begin{tabular}{|c|c|c|}
\hline Id & Strategy & Type \\
\hline HRQ & Highest risk querying & Baseline/BL (Sect. 5.1) \\
\hline $\mathrm{R}$ & Random querying & Exploratory active learning/EAL (Sect. 5.2) \\
\hline $\mathrm{P}$ & Unsupervised (PCA) querying & \\
\hline $\mathrm{U}$ & Uncertainty querying & \\
\hline M & Mix of random and uncertainty querying & \\
\hline SR & SSSL on random points & Stochastic semi-supervised learning/SSSL (Sect. 5.3) \\
\hline SU & SSSL on uncertain points & \\
\hline SM & SSSL on random/uncertain points & \\
\hline SE & SSSL on points most likely to be genuine & \\
\hline SR-U & SSSL on uncertain points + random sampling & SSSL + EAL (Sect. 5.3) \\
\hline SR-R & SSSL on random points + random sampling & \\
\hline SR-M & SSSL on random/uncertain points + random sampling & \\
\hline $\operatorname{SRN}[p]$ & $\mathrm{SR}$ with reduced $\mathrm{x} \%$ of negative feedback & Modified SSSL (Sect. 5.3) \\
\hline ROS & Random oversample & Oversample (Sect. 5.4) \\
\hline SMOTE & SMOTE & \\
\hline $\mathrm{QFU}$ & Querying by frequent uncertainty & Multiple instance learning (Sect. 5.5) \\
\hline MF-... & Max combining function & \\
\hline SM-... & Softmax combining function & \\
\hline LF-... & Logarithmic combining function & \\
\hline
\end{tabular}

